\title{
Endovascular Therapy in Posterior Fossa Brain Arteriovenous Malformations: A Clinical Study
}

\author{
Ozhan Merzuk UCKUN, Ergun DAGLIOGLU \\ Ankara Numune Training and Research Hospital, Department of Neurosurgery, Ankara, Turkey \\ Corresponding author: Ozhan Merzuk UCKUN ozhanmerzuk@gmail.com
}

\section{ABSTRACT}

AIM: To assess posterior fossa brain arteriovenous malformation (PFbAVM) patients who received initial endovascular intervention to reduce the lesion size, as well as embolization for the obliteration of the lesions.

MATERIAL and METHODS: This retrospective analysis included 16 patients ( 7 males and 9 females) with PFbAVMs who were treated between 2010 and 2017 via surgery, endovascular techniques, or combinations of modalities. Patient demographic and clinical features, angiographic characteristics, treatment modalities, complications, and outcomes were analyzed.

RESULTS: Six patients with cerebellar AVMs, 2 with brainstem AVMs, and 8 with cerebellar-brainstem AVMs were included in this study. All patients were treated by endovascular embolization as initial or final treatment. Patient characteristics were assessed for all 16 patients; 7 (43.75\%) presented with hemorrhage, and they were further treated by microsurgery because of residual niduses. Complete obliteration was obtained in all patients. Two patients had persistent neurological deficits. The outcome was measured with the modified Rankin scale (mRS). Serviceable outcome ( $\mathrm{mRS} \leq 2)$ was attained in 14 patients.

CONCLUSION: Successful treatment of PFbAVMs depends on an integrated team of neurosurgeons, radiosurgeons, and endovascular neurosurgeons. In PFbAVMs, endovascular embolization as adjuvant or final treatment is useful and safe.

KEYWORDS: Brain arteriovenous malformation, Endovascular embolization, Neurosurgery, Posterior fossa

\section{INTRODUCTION}

$\mathrm{P}$ osterior fossa brain arteriovenous malformations (PFbAVM) comprise $5-18 \%$ of brain arteriovenous malformations (AVMs). However, PFbAVMs carry a nearly 2-fold greater risk of hemorrhage, compared with other supratentorial equivalents $(2,7)$. Feeding artery aneurysms, deep venous drainage, small lesion size, and age constitute predictive factors for hemorrhage (11). PFbAVMs can be classified by region to organize treatment planning. Lesions may be present on cerebellum (vermis, hemisphere, or combined), brainstem (medulla, pons, or midbrain), or a combination of these 2 regions (3). Presentation differs between supratentorial and infratentorial AVMs. Unlike supratentorial bAVMs, PFbAVMs typically exhibit no symptoms; therefore, they are often diagnosed after hemorrhage occurs, which leads to persistent and severe neurologic findings (10). Reportedly, $72 \%-92 \%$ of patients had at least 1 previous bleeding episode $(2,8)$. Observation, endovascular interventions, surgical resection, radiosurgery, or combinations of these modalities constitute the available treatment options. Most cerebellar AVMs are suitable for microsurgery in which obliteration rates are high (70\%-100\%). Brainstem AVMs are more complicated because of their high mortality and morbidity rates. Therefore, treatment choices for brainstem AVMs are often endovascular techniques and/or radiosurgery (6). The goal of treatment is complete obliteration. Some characteristics, such as eloquent location (e.g., cranial nerves, brainstem structures, or cerebral arteries) and high Spetzler-Martin (SM) grade, hinder total excision via microsurgery. Additionally, complete excision of PFbAVM, particularly those contiguous to important structures, can enhance mortality and morbidity. Notably, 
improvement of endovascular intervention techniques and embolization materials has made endovascular embolization a better choice for PFbAVM treatment. Endovascular embolization can be used as an adjuvant treatment to lower the volume of the nidus before microsurgery or stereotactic radiosurgery; this approach may reduce mortality and morbidity (1). There remains debate regarding the influence of patient age, sex, clinical presentation, lesion location, venous drainage pattern, AVM size, and whether feeding artery-associated aneurysms are involved. We reviewed demographic findings, clinical findings, imaging results, and outcomes of patients with PFbAVM treated between 2010 and 2017 in order to determine the factors associated with treatment modalities, as well as to evaluate the proficiency of endovascular embolization as adjuvant or final treatment.

\section{MATERIAL and METHODS}

Our study was confirmed by the institutional supervision committee and all patients granted informed consent. Sex, clinical presentation, age, lesion location (eloquent or noneloquent), PFbAVM size, deep venous drainage (presence or absence), SM grade, feeding artery aneurysms, treatment modalities, complications, and outcomes were reviewed for all patients. Patients were categorized on the basis of the presence of hemorrhage, according to their clinical presentation. Lesion locations were categorized as eloquent (e.g., major motor pathways, cranial nerves, cerebellum, brainstem, deep cerebellar nuclei, or peduncles) or noneloquent. Venous drainage of PFbAVMs was categorized as deep or superficial (6). The PFbAVM size was classified into 3 groups according to maximum nidus diameter: $<3 \mathrm{~cm}$, 3-6 cm, or $>6 \mathrm{~cm}$. All patients' PFbAVMs were also graded according to the SM grading scale: I to V $(11,13)$ (Table I). Finally, all patients were divided according to the presence of a feeding artery aneurysm, which comprises a sac formed by localized dilatation with a diameter at least 2-fold that of the primary vessel. The mean follow-up period from diagnosis to the final follow-up was 2.3 years.

When determining the treatment modality, considering that demographic risks and concomitant diseases were factors that attenuated the treatment effect, the following aspects were assessed: sex, presentation with hemorrhage or hydrocephalus, age, SM grade, presentation modified Rankin Scale (mRS) score, the presence of deep venous drainage, angiographic features, and lesion location.

Sixteen patients received multimodal treatment, including any combination of surgery and embolization. Microsurgery was performed in patients with hemorrhages because of the risk of compression of the brainstem or fourth ventricle, which can cause hydrocephalus or cerebral hernia. By reduction of bleeding, embolization was performed before microsurgery, with the aim of facilitating the operation, reducing complications, and providing complete elimination of the PFbAVM. For unruptured small PFbAVMs $(<3 \mathrm{~cm})$ with superficial or non-eloquent locations, particularly in young patients, microsurgery was performed to prevent future hemorrhages. The overall goal was complete elimination of the lesion. If total elimination was not possible by one modality alone, multi-modality treatment was considered. Multistage endovascular treatment was appropriate for large PFbAVMs, higher SM grades, and deep locations. Adjuvant endovascular treatment was used before surgery or radiosurgery to lower the risk of persistent neurological impairments. All patients received endovascular treatment as adjuvant therapy $(n=7)$ or multistage embolization as final treatment $(n=9)$. Patients who were referred to an external radiosurgery center because of the absence of the radiosurgery department at our hospital were excluded from the study.

As an index for outcome evaluation, the mRS was used; mRS results were categorized as good outcome (MRS $\leq 2)$ or poor outcome $(\mathrm{MRS} \geq 3)$.

\section{Endovascular Embolization Procedures}

Entire endovascular embolization treatments were applied via transfemoral arterial entry at the same hospital's treatment center by one interventional neuroradiologist and one neurosurgeon with a long-term expertise in endovascular embolization. General anesthesia was administered to the patients and a local antiseptic agent was used for sterilization of the groin. Using a micropuncture set from the right femoral artery, the Neuron (Penumbra, Alameda, CA, USA) guiding sheath was placed with the aid of 80 cm SIM 2125 (Cook Medical, Bloomington, IN, USA). The feeding artery supplying the lesion was superselected using an Apollo microcatheter (Medtronic, Minnesota, MN, USA), Excelsior SL10 microcatheter (Stryker, San Jose, CA, USA), or Mirage micro guidewire (ev3, Irvine, CA, USA). First, to avoid thrombogenesis, systemic heparinization was applied. Continuous saline injection was performed via microcatheter. Under real-time road mapping using digital subtraction angiography (DSA), a 5\% glucose solution was infused from the microcatheter. Coils, stents, and stent-assisted coils were used for embolization. There was no residual aneurysmatic filling on the control angiograms. Right femoral puncture point was closed with 6F Angioseal (Terumo, Somerset, NJ, USA) after right femoral artery catheter extraction. Patients with normal vital signs were extubated. Post-operative care was completed in the intensive care unit. For 48-72 hours, patient blood pressure was maintained at 20 or $30 \mathrm{mmHg}$ lower than normal values. A computerized tomography (CT) scan was performed to detect bleeding or other complications.

\section{Statistical Analysis}

Percentages and frequencies were calculated for categorical variables. Standard deviations (SD) and the mean were calculated for continuous variables. Categorical data were analyzed using the Pearson Chi-Squared test, likelihood test, or Fisher's exact test, as appropriate. Continuous variable analysis was performed using Mann-Whitney U or the Wilcoxon W rank sum test. Patient characteristics such as age, gender, location, clinical presentation, SM grade, and deep venous drainage (presence or absence) were included in univariate analyses. Preoperative and postoperative comparisons of $\mathrm{mRS}$ were performed. A $\mathrm{p}$ value of $<0.05$ was considered significant. The Statistical Package for Social Sciences 13 (SPSS, Chicago, IL, USA) was used for all analyses. 


\section{RESULTS}

In this study, 16 posterior fossa AVM patients were diagnosed and treated via surgery between 2010 and 2017 (mean age 37.06 years, range 11-66 years); 7 (43.75\%) exhibited hemorrhage, 16 (100\%) exhibited headache (including all hemorrhagic patients) and 2 (12.5\%) exhibited focal neurological deficits. The clinical findings, demographic variables, outcome indexes, and angiographic characteristics of AVMs are summarized in Table I.

A total of 21 endovascular embolization procedures were performed. Nine $(56.25 \%)$ patients achieved complete obliteration. Complete obliterations of all lesions were confirmed by DSA after the first and fourth months after endovascular embolization procedures (Figure 1A-G). Seven patients had residual nidus that was treated with microsurgery $(n=7)$ as an additional modality. The duration of follow-up was performed for mean 2.3 years (range 1.1-4.6 years).

Transient complications related to treatment were found in $2(12.5 \%)$ patients: one was due to embolization-induced transient ischemia, the other was due to subarachnoid hemorrhage-induced vasospasm.

Compared with patients treated with endovascular embolization, those treated with surgery or surgery-endovascular embolization combination treatment did not have a better chance of excellent outcome $(\mathrm{mRS} \leq 2)$ on multivariate analysis. The PFbAVM location, size, and clinical grade did not significantly

Table I: Detailed Demographic Characteristics of All Patients Included in the Study. All Features were Compared on the Basis of Patient Outcome

\begin{tabular}{|c|c|c|c|c|}
\hline Demographic Characteristics & $\mathbf{n}$ & $\%$ & $\begin{array}{l}\text { Good outcome } \\
(m R S \leq 2)(n=14)\end{array}$ & $\begin{array}{l}\text { Poor outcome } \\
(m R S>2)(n=2)\end{array}$ \\
\hline Mean age (years) & \multicolumn{2}{|c|}{37.06 (range 11-66) } & 14 & 2 \\
\hline \multicolumn{5}{|l|}{ Sex } \\
\hline Female & 9 & 56.25 & 8 & 1 \\
\hline Male & 7 & 43.75 & 6 & 1 \\
\hline \multicolumn{5}{|l|}{ Presentation } \\
\hline Hemorrhage & 7 & 43.75 & 6 & 1 \\
\hline Headache & 16 & 100 & 14 & 2 \\
\hline Focal neurologic deficits & 2 & 12.5 & 1 & 1 \\
\hline Seizure & 1 & 6.25 & 1 & 0 \\
\hline
\end{tabular}

\section{Lesion location}

$\begin{array}{lcccc}\text { Eloquent (e.g., brainstem, deep cerebellar nuclei) } & 10 & 62.5 & 8 & 2 \\ \text { Non-eloquent } & 6 & 37.5 & 6 & 0\end{array}$

Angiographic features

Size

\begin{tabular}{lrrrr}
\hline$<3 \mathrm{~cm}$ & 1 & 6.25 & 1 & 0 \\
\hline $3-6 \mathrm{~cm}$ & 9 & 56.25 & 8 & 1 \\
\hline$>6 \mathrm{~cm}$ & 6 & 37.5 & 5 & 1 \\
\hline Deep venous drainage & 10 & 62.5 & 8 & 2 \\
\hline Associated aneurysm & 1 & 6.25 & 1 & 0 \\
\hline
\end{tabular}

SM grade

\begin{tabular}{lrrrrr}
\hline I-II & 5 & 31.25 & 5 & 0 \\
\hline III-IV-V & 11 & 68.75 & 9 & 2 \\
\hline
\end{tabular}

Presentation mRankin Score

\begin{tabular}{rrrrr}
\hline$\leq 2$ & 15 & 93.75 & 14 & 1 \\
\hline$>2$ & 1 & 6.25 & 0 & 1 \\
\hline
\end{tabular}




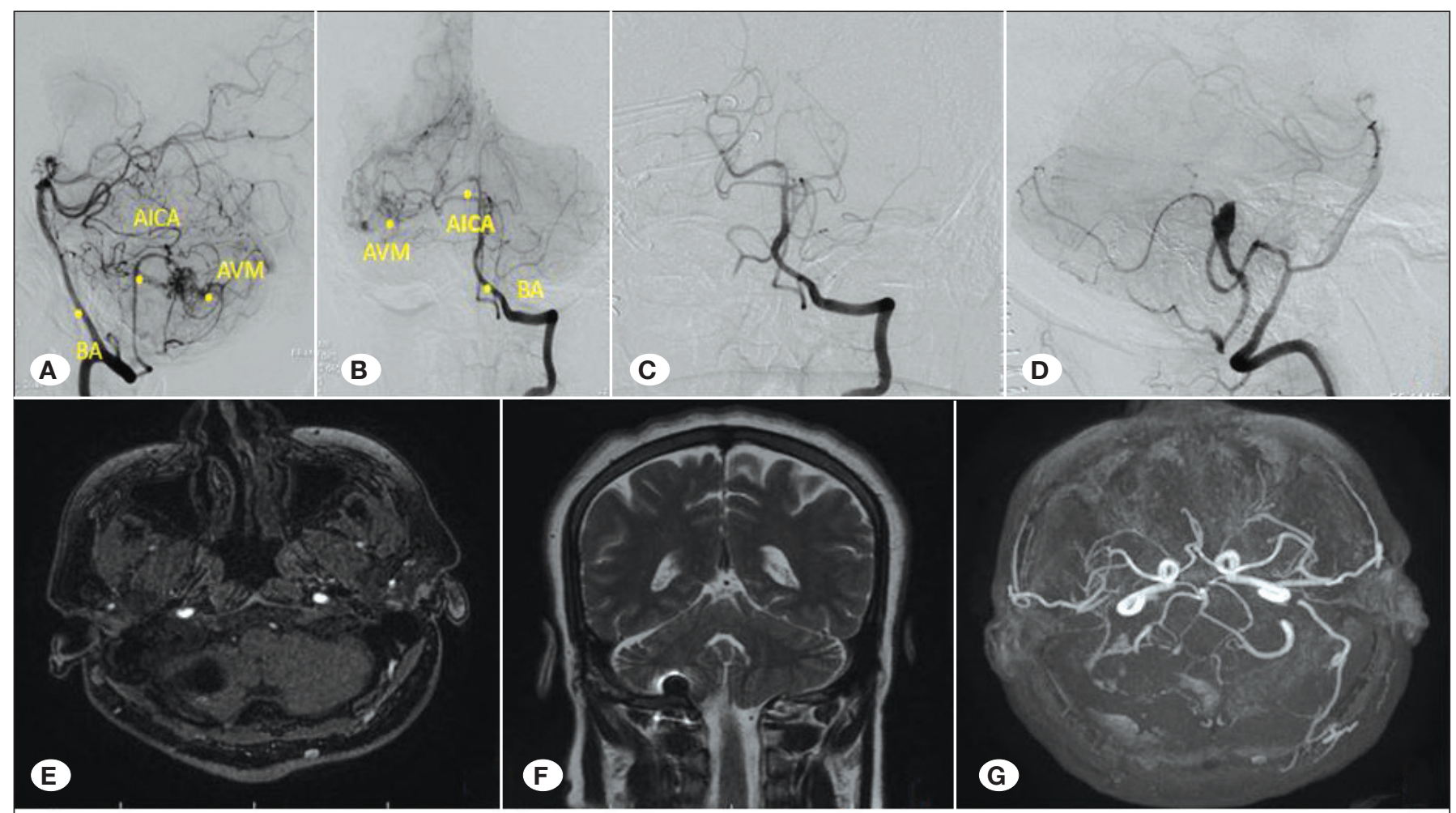

BA: Basilar artery, AICA: Anterior inferior cerebellar artery, AVM: Arteriovenous malformations

Figure 1: Imaging studies of a right-sided cerebellar AVM. Pre-operative DSA images; anteroposterior (A), lateral (B). After embolization+surgery, DSA images (C: lateral) and (D: anteroposterior); and MRI images (E: axial, F: coronal, G: axial) are shown.

affect clinical outcome. Considering patient outcome, hemorrhage at the time of presentation was statistically different between the groups $(p=0.042)$. Among patients with bleeding, localization and deep venous drainage were significantly different $(p=0.037)$, when compared with the corresponding attributes of patients without bleeding.

No significant differences were found in sex ratio, age, related feeding artery aneurysms, number of cases with PFbAVM size less than $3 \mathrm{~cm}$, and low SM grade (grade I and II) between the patient groups with and without hemorrhage. Cerebellar position $(p=0.008)$ and deep venous drainage $(p=0.037)$ were independent predictors of hemorrhage in multivariate analysis.

The demographic and angiomorphologic characteristics of all PFbAVM patients are summarized in Table I. Six (37.5\%) patients had PFbAVM in the cerebellum, 2 (12.5\%) patients had PFbAVMs in the brainstem, and in the remaining patients $(n=8,50 \%)$, lesions were combined. Fifteen (93.75\%) lesions were $\geq 3 \mathrm{~cm}$ and $1(6.25 \%)$ lesion was $<3 \mathrm{~cm}$. Eleven patients $(68.75 \%)$ were of SM grade $\geq \mathrm{III}$; the remaining $5(31.25 \%)$ patients were SM grade $\leq$ II. Ten $(62.5 \%)$ of the patients had deep venous drainage; 6 (37.5\%) had superficial venous drainage. When patient outcomes and mortality-morbidity rates were compared, there were no statistically significant differences between patients treated with final endovascular treatment and those treated with a combination of surgical and endovascular treatment modalities.

\section{DISCUSSION}

PFbAVMs are relatively rare, but have higher rates of morbidity and mortality than supratentorial bAVMs (8). The posterior fossa has a complex anatomy, such that sufficient elimination of PFbAVMs is a necessity. PFbAVMs' relationships with the cerebellum and/or brainstem, cranial nerves, and vascular structures should be visualized before planning a treatment map. Imaging is based upon preoperative DSA, magnetic resonance imaging (MRI) scans, and CT scans.

Based on the anatomy of the posterior fossa, PFbAVMs can be classified into 8 groups (Table II). Brainstem PFbAVMs can be classified as mesencephalic, pontine, or medullary. Also, brainstem PFbAVMs can be classified by the depth of the lesion: pial (superficial) or parenchymal (deep). Pial brainstem PFbAVMs may involve easily controlled vascular structures, such as feeding arteries and drainage veins. Because of their superficial localization, surgery is only recommended in these cases; notably, limited brainstem manipulation capacity can be sufficient for surgery of pial PFbAVM. However, parenchymal (deep) brainstem AVMs are not suitable for surgery, considering their location; they are closely related to important centers, such as cranial nerve nuclei and major motor pathways, and may cause harm to the perforating branches of the main vascular system (4). There is a growing trend of endovascular treatment for these lesions due to the difficulty in performing surgical treatment within this region, 
Table II: Classification of Posterior Fossa Arteriovenous Malformations

\begin{tabular}{cccc}
\hline Name & Location & Arterial supply & Cranial nerves \\
\hline Midbrain & Midbrain & SCA & IV and V \\
\hline Pontine & Pons & AICA/SCA/PICA & VI, VII, VIII \\
\hline Medullary & Medulla & PICA & IX, X, XI, XII \\
\hline Suboccipital & Suboccipital surface of the cerebellum & PICA & IX, X, XI, XII \\
\hline Petrosal & Petrosal surface of the cerebellum & AICA & VII, VIII \\
\hline Tentorial & Tentorial surface of the cerebellum & SCA & IV and V \\
\hline Vermian & & SCAVICA & - \\
\hline Tonsillar & Cerebellar tonsils & PICA & -
\end{tabular}

AICA: Anterior inferior cerebellar artery, PICA: Posterior inferior cerebellar artery, SCA: Superior cerebellar artery.

high mortality and morbidity rates associated with them, as well as the need for skilled surgeons.

Location in the cerebellum and deep venous drainage may be predictors of hemorrhage in patients with PFbAVM (5). The most common site for PFbAVMs is the cerebellum (84.1\%). Also, the cerebellar location may have augmented the risk of high hemorrhage (16). Stefani et al. concluded that a deep bAVM location (eloquent sites, e.g., corpus callosum, basal ganglia, and thalamus) exhibited approximately 5-fold greater risk of bleeding, compared with a superficial location (16).

Patients that presented with hemorrhage showed greater neurological deficit, both before and after surgery (6). Increased bleeding of cerebellar AVMs is not clearly understood $(9,14)$. Furthermore, patients presenting with PFbAVM bleeding have a remarkable re-hemorrhage rate $(8.4 \%-9.4 \%$ per year) (8). These patients also exhibit increased morbidity and mortality rates after re-hemorrhage. Consequently, such patients require more aggressive treatment, as long as the treatment risks are at acceptable levels.

The size of the nidus is associated with an increased risk of rupture. A small nidus has a higher risk of rupture; however, there is no difference in size between cerebellar AVMs and cerebral AVMs. Importantly, cerebellar AVM patients with feeding artery aneurysms had a similar incidence of hemorrhage (9). A notable difference between cerebellar and cerebral AVMs is that patients with cerebral AVMs may present with seizures. Finally, PFbAVM-associated seizures or other apparent symptoms are rare; thus, diagnosis of PFbAVMs is difficult without hemorrhage, and late diagnosis of these lesions contributes to an increased incidence of hemorrhage $(10,15)$.

Complete obliteration is the gold standard for high-risk PFbAVM treatment (11). Microsurgery is considered the best modality for high-risk lesions because of its high obliteration rate; however, the limited and complex surgical area is associated with high mortality and morbidity, due to its proximity to important centers and vascular structures $(6 \%$ and $16 \%$, respectively) $(1,12)$.
In our study, embolization was used both as an adjuvant treatment option and as a final treatment. Multistage endovascular treatment was appropriate for lesions with higher SM grades, large lesions, and those in deep locations. There were no statistically significant differences in mortality and morbidity on the basis of the type of surgery. In 9 patients, multistage embolization was used as the last treatment and complete obliteration was achieved. The other 7 patients showed residual nidus during control DSA; therefore, they underwent surgery as further treatment. Complete obliteration was achieved in all of these patients.

This study has similar limitations to those of other retrospective studies. Our hospital is a tertiary medical center; therefore, this study may include patients whose risk and management is more difficult than typically encountered, which may be a bias in the treatment of patients. In addition, patient sample size and follow-up time led to insufficient statistical outcomes regarding the natural history of the PFbAVMs.

\section{CONCLUSION}

Considering today's evolving embolization techniques and increasing experience, embolization will likely become superior to surgery in lesions with high mortality and morbidity, such as PFbAVMs.

\section{REFERENCES}

1. Almeida JP, Medina R, Tamargo RJ: Management of posterior fossa arteriovenous malformations. Surg Neurol Int 6:31, 2015

2. Al-Shahi R, Warlow C: A systematic review of the frequency and prognosis of arteriovenous malformations of the brain in adults. Brain 124:1900-1926, 2001

3. Batjer H, Samson D: Arteriovenous malformations of the posterior fossa. Clinical presentation, diagnostic evaluation, and surgical treatment. J Neurosurg 64:849-856,1986

4. Batjer HH: Cerebrovascular disease. Philadelphia: LippincottRaven, 1997

5. Choi JH, Mohr JP: Brain arteriovenous malformations in adults. Lancet Neurol 4(5):299-308, 2005 
6. Da Costa L, Thines L, Dehdashti AR, Wallace MC, Willinsky RA, Tymianski M, Schwartz ML, ter Brugge KG: Management and clinical outcome of posterior fossa arteriovenous malformations: Report on a single-centre 15-year experience. J Neurol Neurosurg Psychiatry 80:376-379, 2009

7. Drake CG, Friedman AH, Peerless SJ: Posterior fossa arteriovenous malformations. J Neurosurg 64:1-10, 1986

8. Fleetwood IG, Marcellus ML, Levy RP, Marks MP, Steinberg GK: Deep arteriovenous malformations of the basal ganglia and thalamus: Natural history. J Neurosurg 98:747-750, 2003

9. Fleetwood IG, Steinberg GK: Arteriovenous malformations. Lancet 359:863-873, 2002

10. Garcia MR, Alvarez H, Goulao A, Pruvost P, Lasjaunias P: Posterior fossa arteriovenous malformations. Angioarchitecture in relation to their hemorrhagic episodes. Neuroradiology 31(6):471-475, 1990

11. Khaw AV, Mohr JP, Sciacca RR, Schumacher HC, Hartmann A, Pile-Spellman J, et al: Association of infratentorial brain arteriovenous malformations with hemorrhage at initial presentation. Stroke 35:660-663, 2004
12. Magro E, Chainey J, Chaalala C, Al JH, Fournier JY, Bojanowski MW: Management of ruptured posterior fossa arteriovenous malformations. Clin Neurol Neurosurg 128:78-83, 2015

13. Massager N, Regis J, Kondziolka D, Njee T, Levivier M: Gammaknife radiosurgery for brainstem arteriovenous malformations: Preliminary results. J Neurosurg 93 Suppl 3:102103, 2000

14. Munoz F, Clavel P, Molet J, Castano C, de Teresa S, Solivera J, de Quintana C, Tresserras P, Rodriguez R, Bartumeus F: Current management of arteriovenous malformations. Retrospective study of 31 cases and literature review. Neurocirugia (Astur) 18(5):394-404, 2007 (in Spanish)

15. Rodriguez-Hernandez A, Kim H, Pourmohamad T, Young WL, Lawton MT: Cerebellar arteriovenous malformations: Anatomic subtypes, surgical results, and increased predictive accuracy of the supplementary grading system. Neurosurgery 71(6):1111-1124, 2012

16. Stefani MA, Porter PJ, TerBrugge KG, Montanera W, Willinsky $\mathrm{RA}$, Wallace MC: Large and deep brain arteriovenous malformations are associated with risk of future hemorrhage. Stroke 33(5):1220-1224, 2002 